\title{
Scale-independent Indicators and Research Evaluation
}

\author{
J. Sylvan Katz \\ SPRU, \\ Science and Techonology Policy Research \\ University of Sussex \\ Brighton, E. Sussex \\ UK BN1 9RF \\ j.s.katz@sussex.ac.uk
}

forthcoming

Science and Public Policy

06 March 2000 


\title{
Scale-independent Indicators and Research Evaluation
}

J. Sylvan Katz

\begin{abstract}
Does the size of institution or system affect the amount of recognition it receives, the impact it has on others and the degree to which it collaborates? Is it possible to optimise size to maximise recognition, impact and co-operation? This paper demonstrates that some conventional indicators used in research evaluation may fail to account for the non-linearity between size and performance. This can result in an over- and under-estimation of the research performance of both large and small institutions and nations. This paper shows that a power law relationships exists between recognition or impact and (a) the publishing size of scientific communities within an OECD science system and (b) the publishing size of a research community across OECD science systems or institutions in a science system. Also, a power law relationship exists between the amount of various types of collaboration and the publishing size of institutions. It also shows that there are power law relationships between publishing size and HERD or number of researchers. The exponent of the power law is sometimes greater than 1.0 indicating the existence of a "Matthew effect". Other times it is less than 1.0 indicating an "inverse Matthew effect". A power law is the common signature of a scale-independent process that can be typified by a geometric fractal and other self-similar properties. A new class of scale-independent indicators is developed to overcome the inequity produced by some non-linear characteristics commonly measured when evaluating research performance.
\end{abstract}




\title{
Scale-independent Indicators and Research Evaluation
}

\author{
J. Sylvan Katz \\ SPRU, University of Sussex, Brighton, E. Sussex, UK BN1 9RF \\ j.s.katz@sussex.ac.uk
}

\begin{abstract}
A very common view is that these scaling or power-law relations are nothing more than the simplest approximation to the available experimental data, having no special advantages over other approximations. It is not so. Scaling laws give evidence of a very deep property of the phenomena under consideration - their self-similarity: such phenomena reproduce themselves, so to speak, in time and space. [Barenblatt, 1997 p. xi]
\end{abstract}

Have you ever wondered how the size of a group, an institution or system affects the amount of recognition it receives, the impact it has on others and the degree to which it collaborates with other groups, institutions or systems? Do you think it is possible to optimise the size of a group, institution or system to maximise its recognition, impact and collaboration? Is it important to consider the size of a group, institution or system in comparative evaluation activities? Can we develop policies to shape the impact, recognition and collaborative characteristics of a group, institution or system? Questions of this nature continually arise in evaluation activities and policy formulation. In fact they probably have contributed to the increasing movement toward evidence-based evaluation and policy.

This paper endeavours to provide some insight into these questions by exploring indicators that measure the performance and activities of the scientific community. The evidence will be provided using measurements ${ }^{1}$ of size, recognition, impact and collaboration derived from one of the most common products of scientific activity - the research paper. Size is measured by counting refereed scientific publications. Impact and recognition are measured using citations to these papers. And collaboration is measured using information derived from the institutional addresses listed on co-authored publications. Recognition and impact will be compared to size across scientific communities within a nation and across nations within scientific fields. The amount of various types of institutional collaboration will be compared to institutional size within the UK science system. And size will be compared to HERD ${ }^{2}$ and numbers of researchers for OECD countries.

It will be demonstrated that in each instance a power law or scale-independent relationship can describe the non-linear relationship between the variables ${ }^{3}$. We will show that conventional indicators used in research evaluation usually fail to account for this nonlinearity and frequently over estimate or under estimate the research performance of large and small groups, institutions and nations. And finally we will develop a new class of scaleindependent indicators that may provide a more equitable portrait of the similarities and differences among research performers of varying size.

\section{Introduction}

Invariably an evaluation of national and international scientific activity includes bibliometric measures of

- size - number of published papers,

- recognition - number of citations to papers,

- impact - citations/paper,

- collaboration - number of co-authored papers.

\footnotetext{
These measures are called bibliometric indicators. An extensive discussion of the advantages and limitations of these types of indicators is given in Katz and Hicks (1997b).

HERD = Higher Education Expenditure on R\&D

A power law relationship is described by $\mathrm{y}=\mathrm{ax}^{\mathrm{n}}$ where $\mathrm{a}$ is a constant and $\mathrm{n}$ is a real number.
} 
The advantages and limitations of these measures are thought to be well understood (Katz and Hick, 1997a). It is widely believed that the number of papers published by a group, institution or nation is a partial indicator of its size and that the impact of the research is a partial measure of the research quality and it is independent of size. In fact the relative citation impact indicator (RCI) that is derived directly from the measures of size and recognition and will be discussed in detail later is used to compare the research impact of groups, institutions and nations in a scientific field. For example, Robert May (e.g. 1997) used the RCI to support his argument that better value for money might be associated with performing basic research in universities rather than in research institutions. Also, the HEFCE-commissioned bibliometric study of English HEIs compared the RCI to scores received in the research assessment exercise (Adams et al., 1998).

The RCI of a scientific community in a country is determined by taking the ratio between the impact of the publications in the field that involve authors from the country of interest and the impact of all publications in the field. It is given by the following relationship

$$
\mathrm{RCI}_{\mathrm{i}}=\text { country }_{\mathrm{i}} \text { impact/world impact }=\mathrm{I}_{\mathrm{i}} \mathrm{I}_{\mathrm{w}}
$$

where impact $I$ = citations/paper. A similar measure is used to compare groups and institutions. Traditionally the impact indicator is considered to be robust. Its use is based on the assumption that the number of citations a collection of papers receives is independent of the collection size. Generally, the RCI is only used to compare the impact of national science systems in a given research field. It is rarely, if ever, used to compare the impact of different research fields within a science system or across national science systems. The reason for this is that it is assumed that the citation patterns and culture vary from field to field thus making impact incomparable across research areas. However, the assumption that the number of citations is independent of publishing size may be incorrect. In fact, there may be a strong relationship between the size of a group, institution and nation and the amount of recognition it receives, the impact it has on others and the degree to which it collaborates with other groups, institutions or nations.

This paper will show that the recognition and impact of an institution, community or nation and the amount of institutional collaboration in a national science system increases in a power law relationship or scale-independent ${ }^{4}$ manner with the size of the institution, community or nation. It will suggest that these scale-independent phenomena probably extend all the way from the level of the research group, and perhaps the individual, through to the level of the institution, nation and the global scientific enterprise. It advocates that research evaluation must develop new indicators that adjust for effect of size on recognition, impact and collaborative activities when comparing the performance of groups, institutions and nations.

\section{Recognition and size}

In two recent papers the author showed that a power law relationship ${ }^{5}$ existed between recognition (citations) and size (papers) across:

a) scientific communities ${ }^{6}$ within a science system (Katz, 1999a)

b) science systems for a specific scientific community (Katz, 1999b)

And in this paper it will be shown that the relationship also holds across:

c) institutions in various sectors within a science system.

4 Given $y=f(x)$ and $y^{\prime}=f(s x)$ then a scale-independent relationship is one that satisfies $y / y^{\prime}=s^{n}$.

5 The power law is given by $\mathrm{c}=\mathrm{kp}^{\mathrm{n}}$ where $\mathrm{c}$ is citations, $\mathrm{p}$ is papers and $\mathrm{k}$ is a constant. A linear relationship (i.e. $\mathrm{n}=1$ ) is a special case of a power law.

6 A community is defined as a group of researchers that publishes papers in the same set of journals (see Katz, 1999a for details) 
Let us examine each of case separately.

\subsection{Recognition and the size of scientific communities within a science system}

Figure 1 illustrates the power law relationship between recognition and size for scientific communities ${ }^{7}$ within the world science system. It is a plot of refereed papers ${ }^{8}$ indexed in the Science Citation Index (SCI) between 1981 and 1992 and the citations received by those papers between 1981 and 1994 counted using a fixed 3-year citation window ${ }^{9}$. The relationship observed between citations, $c$, and papers, $\mathrm{p}$, is given by equation 2

where $\mathrm{k}$ is a constant.

$$
\mathrm{c}=\mathrm{kp}^{1.27 \pm 0.03}
$$

Also, it has been demonstrated (Table 2) that the exponent of the power law relationship was relatively constant over time and it is reasonably constant for a number of OECD science systems and institutional sectors within the UK science system [Katz, 1999a]. Although not identical the national exponents are similar to the world exponent and have $\mathrm{R}^{2}$ values between 0.89 and 0.94 . This indicates that the relationship between the amount of recognition and the size of scientific communities within a science system is scale-independent i.e. there is no maximum size for a scientific community that will produce a optimum amount of recognition. In fact these communities are part of a self-similar national system that in turn is part of the self-similar or fractal global scientific enterprise (Katz, 1999a).

Table 1 - Exponent stability of power law relationship between recognition and size in world science

\begin{tabular}{ccc}
\hline Year & Exponent & $\mathbf{R}^{2}$ \\
\hline 1981 & $1.25 \pm 0.02$ & 0.92 \\
1982 & $1.26 \pm 0.03$ & 0.92 \\
1983 & $1.26 \pm 0.03$ & 0.92 \\
1984 & $1.24 \pm 0.03$ & 0.91 \\
1985 & $1.26 \pm 0.03$ & 0.92 \\
1986 & $1.26 \pm 0.03$ & 0.91 \\
1987 & $1.27 \pm 0.03$ & 0.91 \\
1988 & $1.27 \pm 0.03$ & 0.91 \\
1989 & $1.27 \pm 0.03$ & 0.91 \\
1990 & $1.28 \pm 0.03$ & 0.91 \\
1991 & $1.24 \pm 0.03$ & 0.91 \\
1992 & $1.26 \pm 0.03$ & 0.91 \\
\hline
\end{tabular}

Recall we are using citations as a partial indicator of scientific recognition. The data also suggest that there is a strong "Matthew effect"10 (Merton, 1968). Essentially this means a scientific community experiences a non-linear increase in the amount of recognition it receives as its size increases. For example, in the world or UK science system each time the

7 A scientific community corresponds to one of the 152 ISI subject categories (see Katz, 1999a for details).

8 These data were purchased from ISI for a NERC project. Only article, note or review publication types were counted

9 Current year of publication plus the subsequent two years

${ }^{10}$ Robert K. Merton named the Matthew Effect, after the Gospel According to St. Matthew: "For unto every one that hath shall be given, and he shall have abundance; but from him that hath not shall be taken away even that which he hath." 
Figure 1 - Recognition and size in world science (1981-1992)

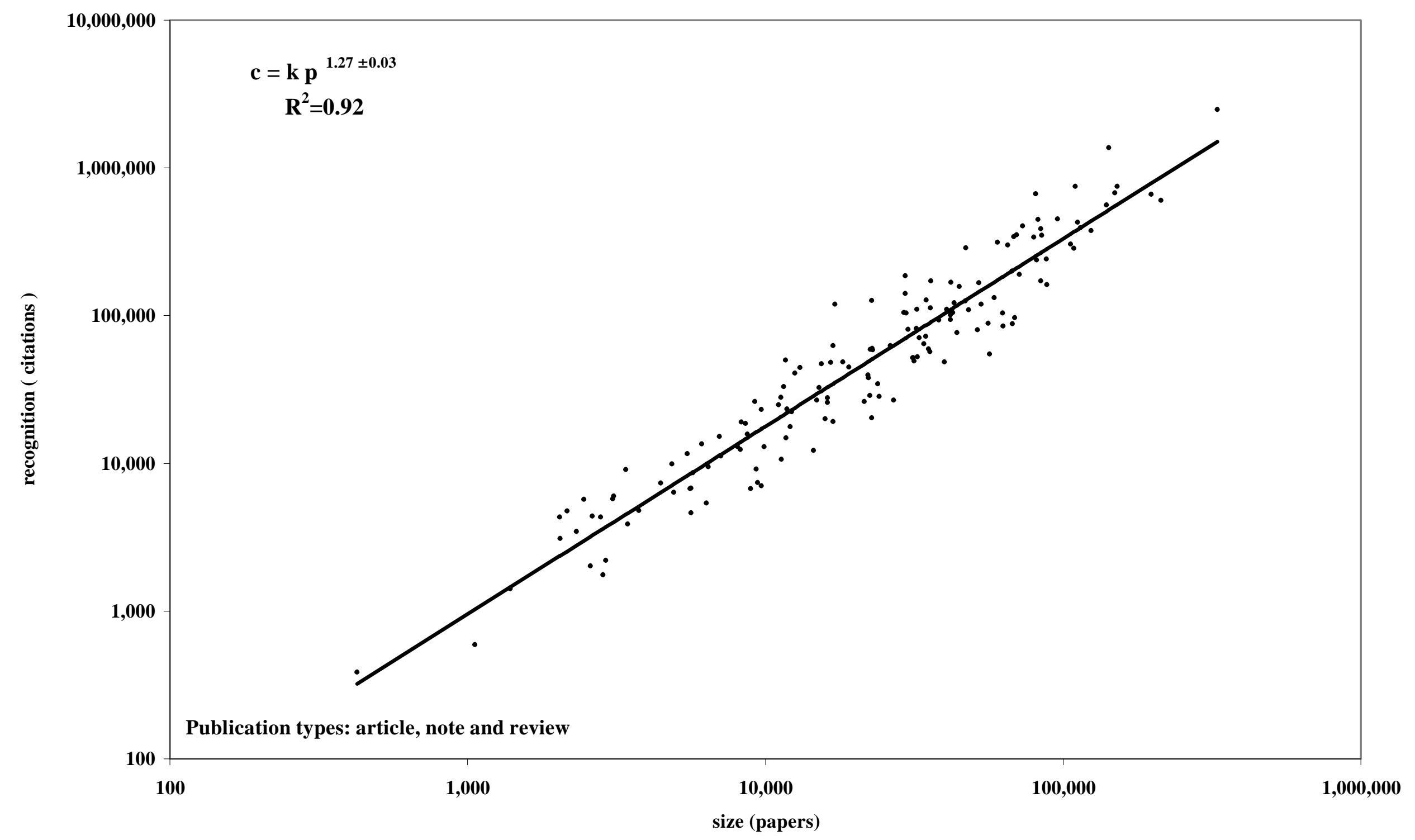


size doubles on average the amount of recognition increases by $2.41 \pm 0.05$ times (i.e. $2^{1.27 \pm 0.03}$ ) and in the US system it increases by $2.53 \pm 0.07$ times (i.e. $2^{1.34 \pm 0.04}$ ). Size does matter; it affects recognition.

Table 2 - Exponent of power law relationship between recognition and community size for various OECD countries

\begin{tabular}{lccc}
\hline Country/sector & $\begin{array}{c}\text { Number of } \\
\text { communities }\end{array}$ & Exponent & $\mathbf{R}^{\mathbf{2}}$ \\
\hline World & 152 & $1.27 \pm 0.03$ & 0.92 \\
EU & 152 & $1.25 \pm 0.02$ & 0.94 \\
US & 152 & $1.34 \pm 0.04$ & 0.89 \\
UK & 152 & $1.27 \pm 0.03$ & 0.93 \\
France & 152 & $1.22 \pm 0.02$ & 0.95 \\
Germany & 152 & $1.23 \pm 0.03$ & 0.93 \\
Canada & 152 & $1.25 \pm 0.03$ & 0.90 \\
Australia & 152 & $1.20 \pm 0.03$ & 0.91 \\
\hline UK sector & 152 & $1.23 \pm 0.03$ & 0.93 \\
$\quad$ Education & 103 & $1.23 \pm 0.03$ & 0.95 \\
$\quad$ Medical & 137 & $1.16 \pm 0.05$ & 0.81 \\
$\quad$ Industry & 145 & $1.28 \pm 0.03$ & 0.90 \\
\hline \multicolumn{1}{c}{ Other } & & &
\end{tabular}

\subsection{Recognition and the size of a scientific community in different systems}

Figure 2 illustrates that a power law relationship exists between recognition and size for six scientific communities ${ }^{11}$ calculated across national science systems. Only those science systems that published at least 10 papers between 1994 and 1998 in the field were used in the analysis. These data were derived from the deluxe version of ISI ${ }^{\circledR}$, s National Science Indicators on Diskette (NSIOD) version 1.5. Figure 2 is a plot of refereed papers ${ }^{12}$ indexed in various ISI databases ${ }^{13}$ between 1994 and 1998 and the citations received by those papers between 1994 and 1998 counted using a variable 5-year citation window ${ }^{14}$. The power law relationship observed between recognition and size in these instances have exponents that range from 1.14 to 1.21 and they have $\mathrm{R}^{2}$ values that range from 0.91 to 0.95 . Again the "Matthew effect" can be quite strong witnessed by the fact that on average the amount of recognition can increase by 2.2 to 2.3 times if the size of the community is doubled.

\subsection{Recognition and the size of an institution in a national science system}

Table 3 gives the exponent for the power law relationship between recognition and institutional size calculated for institutions in various UK sectors and scientific disciplines.

11 The communities were defined using the ISI Current Contents fields: physics; biochemistry \& biophysics; electrical \& electronic engineering; biotechnology \& applied microbiology; molecular biology \& genetics; pharmacology \& toxicology (see http://www.isinet.com for definitions of these fields)

12 ISI defines a refereed paper as an article, note, review or conference proceeding publication type.

${ }^{13}$ For example, the Science Citation Index (SCI), the Social Science Citation Index (SSCI), Arts \& Humanities Citation Index (AHCI).

14 This is way that ISI typically counts citations. It is not as accurate as the fixed citation window counting technique since publications earlier in the interval accumulate more citations than those published at the end of the interval. For example, in the case under discussion papers published in 1994 accumulate citations from 1994 to 1998 while papers published in 1998 will receive few, if any citations, in 1998. 
Figure 2 - Recognition and size across national science systems (1994-1998)
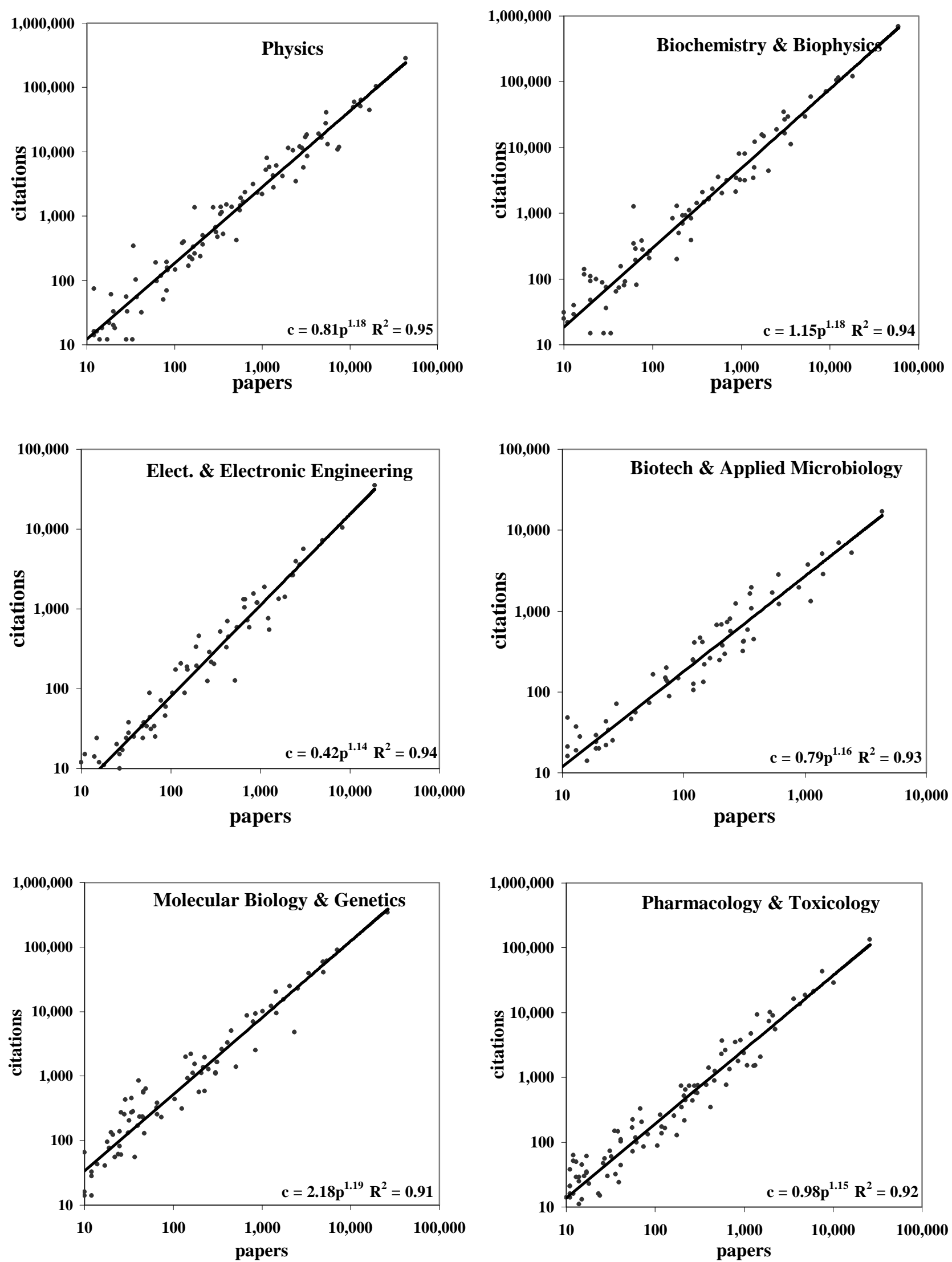
The data were derived from the BESST ${ }^{15}$ database [Katz and Hicks, 1997]. The BESST database consists of papers indexed in the SCI between 1981 and 1994 that listed at least one UK institutional address. Each UK institutional name was unified to one of approximately 6,700 standard institutional names and each institution was assigned to one of six institutional ${ }^{16}$ sectors. A journal classification scheme was used to assign papers to one of four scientific disciplines [Katz and Hicks, 1995]: life sciences, natural sciences, engineering \& material sciences, and multidisciplinary sciences. In this analysis a fixed three-year window was used to count citations. Also only institutions that published at least one paper each year between 1981 and 1994 and received at least one citation within the three-year citation window were used.

Table 3 - Exponent of the power law relationship between recognition and institutional size for various scientific disciplines

\begin{tabular}{lcccc}
\hline \multirow{2}{*}{ Discipline } & UK Sector & $\begin{array}{c}\text { Number } \\
\text { Institutions* }\end{array}$ & Exponent & $\mathbf{R}^{\mathbf{2}}$ \\
\hline All disciplines & All & 483 & $1.13 \pm 0.02$ & 0.91 \\
& Education & 97 & $1.21 \pm 0.02$ & 0.98 \\
& Medical & 230 & $1.19 \pm 0.02$ & 0.95 \\
Life sciences & Industry & 55 & $1.22 \pm 0.07$ & 0.84 \\
& All & 426 & $1.15 \pm 0.02$ & 0.91 \\
& Education & 83 & $1.15 \pm 0.02$ & 0.97 \\
Natural sciences & Medical & 229 & $1.18 \pm 0.02$ & 0.95 \\
& Industry & 29 & $1.25 \pm 0.08$ & 0.89 \\
& All & 131 & $1.12 \pm 0.02$ & 0.94 \\
& Education & 82 & $1.22 \pm 0.02$ & 0.98 \\
Engineering \& material sciences & Medical & 2 & - & - \\
& Allustry & 18 & $1.18 \pm 0.11$ & 0.89 \\
& Education & 54 & $1.12 \pm 0.05$ & 0.91 \\
& Medical & 1 & $1.14 \pm 0.06$ & 0.91 \\
Multidisciplinary sciences & Industry & 6 & $1.31 \pm 0.09$ & 0.98 \\
& All & 123 & $1.00 \pm 0.08$ & 0.58 \\
& Education & 63 & $1.22 \pm 0.07$ & 0.84 \\
& Medical & 16 & $1.05 \pm 0.30$ & 0.47 \\
& Industry & 10 & $1.49 \pm 0.28$ & 0.78 \\
\hline
\end{tabular}

* the number of UK institutions that published at least one paper each year between 1981-1994 receiving at least one citation.

Note that relatively few medical institutions published papers in the natural and engineering $\&$ materials sciences and therefore the exponent was not computed for these cases. Again the power law relationship between size and recognition is evident and the Matthew effect is quite strong. On average if an institution in the UK science system doubles its size it can expect its recognition to increase by 2.19 times (i.e. $2^{1.13 \pm 0.02}$ ). However, if a medical institution doubles its publishing size in the life sciences it can expect to increase its recognition on average by 2.27. A doubling in the size of an educational institution's publishing size in the natural sciences can earn it an average increase in recognition of 2.33 times. To put this in another context, the University of Sussex published 2,370 papers in the natural sciences between 1981 and 1994 while the University of Cambridge published 9,275

\footnotetext{
15 BESST - Bibliometric Exploration of Sectoral Scientific Trends

16 The institutional sectors are education, medical, government, research councils, industry and non-profit (see Katz and Hicks, 1997 for details).
} 
or 3.9 times as many papers. On average Cambridge should expect to receive 5.2 times $\left(3.9^{1.22 \pm 0.02}\right)$ as many citations as Sussex. In fact it received 5.0 times as many citations indicating that the University of Sussex was doing better than expected given its size. In comparison the conventional impact indicator shows that while Cambridge earned 5.71 citations/paper the University of Sussex received 4.51 citations/paper. Given that size can affect impact how should we measure the impact of a group, institution or nation's research effort? We will look at this question shortly.

\section{Impact and size}

Traditionally impact is the basic bibliometric indicator that is used in bibliometric comparisons of research performance. The impact of a collection of papers is determined by taking the ratio between the amount of recognition (citations) received by the collection and the size of the collection (papers). In other words the impact, I, is given by

$$
\mathrm{I}=\mathrm{c} / \mathrm{p}
$$

where $\mathrm{p}$ is papers and $\mathrm{c}$ is citations. While impact is not a direct or absolute measure of the average quality of the research in the collection it has been shown to exhibit a significant correlation with the quality.

The value of the impact measure is based on the assumption that recognition and size are independent variables and that the resultant impact measure is independent of size. However, we can see from the cases that we have examined that this assumption is wrong. Papers and citations are not independent variables. For example, using equation 2 from case (a) we can derive equation 4

$$
\mathrm{I}=\mathrm{c} / \mathrm{p}=\mathrm{kp}^{0.27 \pm 0.03}
$$

This equation indicates that the impact increases in a power law relationship to the numbe of published papers. Impact is not normalised for publishing size. In fact it varies in a scaleindependent manner with publishing size. As mentioned earlier, the traditional comparative measure of impact is the relative citation impact (RCI) given by equation 1 . Since this measure is derived directly from the impact measure we can conclude that it also varies in a power law relationship with size.

\section{Collaboration and size}

Research collaboration appears to be the rule and not the exception. For example, by 1995 $50 \%$ of all scientific papers involved 2 or more authors and 30\% involved institutions in 2 or more countries ${ }^{17}$. In $199488 \%$ of all UK education papers involved two or more authors and $55 \%$ involved two or more institutions. They collaborated with other domestic institutions on $34 \%$ of their papers and with international partners on $20 \%$ of their papers. But is there a relationship between collaboration and size? Is the Matthew effect also at work in cooperative research too?

Using data for UK educational ${ }^{18}$ institutions derived from the BESST database the relationship between the publishing size of an institution and the frequency of occurrence of various types of collaboration was investigated. Collaborations were determined using the information given in the corporate address field of each paper indexed in the SCI and they were classified into various types given in table 4 .

\footnotetext{
${ }^{17}$ Source: National Science Board's Science \& Engineering Indicators, 1998, Table 5-2

${ }^{18}$ A UK educational institution is defined as an 'old' university, 'new' university (polytechnic) or other educational institution.
} 
Table 4 - Definitions of collaboration types

\begin{tabular}{ll}
\hline Collaboration type & Definition \\
\hline multiple author & papers involving two or more authors \\
domestic & papers involving two UK institutions \\
international & papers involving a UK and at least one non-UK institution \\
intra-institution & papers involving two departments in the same institution \\
intra-sectoral & papers involving two or more institutions in the same sector \\
inter-sectoral & papers involving institutions two different institutional sectors \\
industry & papers involving at least one UK industrial partner \\
\hline
\end{tabular}

Collaborative papers were "whole counted", meaning that the data were based on counts of papers that list an address from at least one institution in that sector ${ }^{19}$. The counts were interpreted as the number of papers in which a sector or institution participated. This is a straightforward and intuitive method of interpreting publication counts in an age of increasing collaboration. This method does not involve auxiliary, unproven assumptions about the amount of work represented by authorship on a paper and it is a method that facilitates analysis of this most important component of scientific output by making it visible.

Only collaboration for UK educational institutions that published at least one paper each year during the fourteen-year time interval were examined in this analysis. Ninety-six institutions met this criterion. A power law relationship was found between the amount of collaboration of various types and the size of the institutions. The results are summarised in Table 5 and they are ordered in ascending order of the value of the exponent.

The amount of collaboration shows a scale-independent relationship with institutional publishing size. However, unlike recognition and impact the amount of collaboration does not always exhibit a "Matthew effect". In fact, it appears that smaller educational institutions have a greater propensity than larger institutions to collaborate domestically, particularly with industrial partners and other educational institutions (i.e. exponent $<1.0$ ). On the other hand larger institutions have a greater propensity than smaller institutions to collaborate internally and internationally (i.e. exponent $>1.0$ ). Inter-sectoral and multiple author collaborations tend to increase linearly with size (i.e. exponent $=1.0$ ).

Table 5 - Exponent for power law relationship between collaboration of various types and institutional size

\begin{tabular}{lcc}
\hline Collaboration type & Exponent & $\mathbf{R}^{\mathbf{2}}$ \\
\hline Industry & $0.81 \pm 0.04$ & 0.82 \\
Intra-sectoral & $0.82 \pm 0.02$ & 0.94 \\
Domestic & $0.89 \pm 0.02$ & 0.96 \\
Inter-sectoral & $0.99 \pm 0.03$ & 0.94 \\
Multiple author & $1.02 \pm 0.01$ & 1.00 \\
International & $1.14 \pm 0.03$ & 0.95 \\
Intra-institutional & $1.32 \pm 0.05$ & 0.86 \\
\hline
\end{tabular}

Using the dat ${ }^{20}$ for 40 countries from the National Science Board's Science \& Engineering Indicators (1998) we explored the relationship between multiple author collaborations and national size and international collaborations and national size for 40 countries. Between 1981

\footnotetext{
${ }^{19}$ Alternatively, papers could be fractionated among participating institutions. In this case, if there were two addresses listed on a paper, each institution would be credited with half a paper.

${ }^{20}$ National Science Board's Science \& Engineering Indicators, 1998, Table 5-2
} 
and 1995 the exponent for the power law relationship between multiple author collaborations and size increased slightly from $0.95 \pm 0.03\left(\mathrm{R}^{2}=0.97\right)$ to $0.99 \pm 0.02\left(\mathrm{R}^{2}=0.99\right)$,

respectively. In essence the relationship is quite linear and stable. On the other hand the exponent for relationship between international collaborations and size changed from $0.85 \pm$ $0.03\left(\mathrm{R}^{2}=0.97\right)$ to $0.91 \pm 0.02\left(\mathrm{R}^{2}=0.98\right)$ in the same time period. At the beginning of the period smaller countries had a greater propensity to collaborate than larger countries. This non-linearity has decreased somewhat with time but it still exists. Again we see the "inverse Matthew effect" at work.

\section{Summary}

It has been demonstrated that recognition and impact increase in a power law relationship with the size of an institution, community or nation. Collaboration exhibits a power law relationship with the size of an institution. In other words these characteristics are scaleindependent. They do not exhibit a maximum at some optimum size. Recognition and impact appear to exhibit a "Matthew effect" demonstrated by the fact that the exponent of the power law is greater than 1.0 in the examples that have been presented here. On the other hand while some types of collaboration exhibit a "Matthew effect" others types exhibit the inverse effect.

Before we address the question of how to construct an impact indicator that is properly adjusted for size let us consider the factors that govern the magnitude of the exponent in the power law relationships.

\section{Factors governing the magnitude of a power law exponent}

At the simplest level of interpretation the exponent of the power law is a measure of the linearity of the relationship between the dependent and independent variables - in this case the relationship between recognition, impact, collaboration and size. If the exponent is equal to 1.0 then there is a linear relationship between the variables. If the exponent is less than 1.0 then the dependent variable does not increase as rapidly as the independent variable. For example a doubling in size produces less than a doubling in the amount of recognition. If the exponent is greater than 1.0 the dependent variable increases faster than the independent variable. For example, a doubling in size produces more than a doubling in the amount of recognition.

What factors influence the magnitude of the exponent? We could ask what factors influence size and what factors besides size affect recognition, impact and collaboration. The dilemma we face using this approach is that all of these characteristics are influenced by many common factors. For example, funding is a fundamental factor that can influence each of these. Money can be used to increase the size of a research group making it possible for it to increase its published output. It can be used to purchase new instruments allowing an existing research group to perform higher impact research. Or the funds could be used to collaborate with another group of highly skilled and productive researchers. Not only would this increase the effective size of the group but could increase its recognition and impact. In fact not only can size directly affect recognition, impact, and collaborative activity but each of these can also feed into the peer review process and affect size as well as each other. In essence, the exponent is a measure of the complex interplay among all the economic, social, political and cultural factors that influence the size and recognition, impact or collaboration. These factors include but are not limited to such things as funding, national and international research priorities, institutional structure, language, culture, social concerns, etc. The list is endless. Perhaps, all that can be said is that the exponent emerges from the dynamic interplay among all the factors that influence interdependent pairs of variables. 


\section{How can we adjust for the effect of size on recognition and impact?}

The power relationship between citations and papers gives us a tool for developing a new type of indicator that takes into consideration the effect of size. For example, the power law relationship between citations, $\mathrm{c}$, and papers, $\mathrm{p}$, is given by the general equation

$$
\mathrm{c}=\mathrm{kp}^{\mathrm{n}}
$$

where $\mathrm{n}$ is the slope of the $\log$-log regression line and $\mathrm{k}$ is a constant. Using this relationship we can calculate the expected number of citations, $\mathrm{c}_{\mathrm{e}}$, that should be received for the publishing size, $\mathrm{p}$, and the value of the exponent, $\mathrm{n}$, for the system under consideration. Now using $\mathrm{c}_{\mathrm{e}}$ we can calculate the expected impact, $\mathrm{I}_{\mathrm{e}}$, as follows

$$
\mathrm{I}_{\mathrm{e}}=\mathrm{c}_{\mathrm{e}} / \mathrm{p}=\mathrm{kp} \mathrm{p}^{\mathrm{n}-1}
$$

The conventional or actual impact, $\mathrm{I}_{\mathrm{o}}$, is given by

$$
\mathrm{I}_{\mathrm{o}}=\mathrm{c}_{\mathrm{o}} / \mathrm{p}
$$

where $\mathrm{c}_{\mathrm{o}}$ is the observed number of citation received for $\mathrm{p}$ papers. The ratio between the observed impact and the expected impact gives us a measure of how much impact a field is actually having compared to the amount of impact it is expected to have given its publishing size. Let us call this ratio the adjusted relative citation impact (ARCI) and it is given by as follows:

$$
\mathrm{ARCI}=\mathrm{I}_{\mathrm{o}} / \mathrm{I}_{\mathrm{e}}=\mathrm{c}_{\mathrm{o}} / \mathrm{c}_{\mathrm{e}}
$$

By way of an example consider the first case that was presented. In that case we examined the relationship between recognition and size for various communities within a science system (see Figure 1). Table 6 gives the RCI values and the ARCI for the top twenty traditional high impact ISI science fields ${ }^{21}$. The ARCIs for each country were calculated using the exponent of the power law relationship between recognition and size for that country (e.g. for the UK an exponent of 1.25 was used and for the US an exponent of 1.34 was used). It is worth mentioning that the ARCI has an additional advantage. Since we have adjusted the impact to compensate for the effect of size we can now make comparisons both among fields within and among systems. In other words, we can compare (a) fields of different sizes within the same system and (b) the same field across systems. In fact, in case (b) we are comparing the impact of a community of a specific size given the nature of the system in which the community resides. This occurs because we use the exponent for a given system to compute the ARCI and as explained earlier the exponent has embedded in it characteristics of the system from which it was derived as well as the characteristics of the environment in which the system interacts.

The picture that is portrayed using the ARCI is different from the one we are used to seeing using only the $\mathrm{RCI}^{22}$. According to the RCI indicator the US dominates world science; it has the highest RCI in 70 of the 152 fields. However, according to the ARCI the US ranks highest in only 16 fields while Germany and France rank highest in 35 fields. Interestingly, the highest ARCI in the table belongs to Germany in multidisciplinary sciences. In comparison to the RCI indicator, the ARCI indicator shows the UK maintained its lead in biophysics, shared its high rank in neurosciences with France, lost its highest rank in oncology to Australia but gained the highest rank in developmental biology from the US.

21 There are 152 fields in total

${ }^{22}$ It is important to note that since the data that were available for this study does not cover all countries in the world a comparison could only be made between those countries for which data was available. If Japan, other European and Eastern European countries are included the number of fields in which France and Germany lead according to the ARCI values will decrease significantly. 


\begin{tabular}{|c|c|c|c|c|c|c|c|c|c|c|c|c|c|c|c|c|}
\hline \multirow[b]{2}{*}{ Field } & \multirow{2}{*}{$\begin{array}{l}\begin{array}{l}\text { World } \\
\text { (c/p) }\end{array} \\
\end{array}$} & \multicolumn{7}{|c|}{ RCI } & \multicolumn{8}{|c|}{$\overline{\text { ARCI }}$} \\
\hline & & US & EU & UK & France & Germany & Canada & Australia & World & US & EU & UK & France & Germany & Canada & Australia \\
\hline Multidisciplinary Sciences & 9.61 & $\mathbf{1 . 8 5}$ & 1.14 & 1.34 & 0.85 & 1.41 & 1.29 & 1.00 & 2.63 & 3.51 & 3.30 & 3.33 & 0.84 & 4.53 & 3.95 & 3.18 \\
\hline Cytology \& Histology & 8.27 & 1.58 & 0.84 & 1.10 & 0.81 & 1.03 & 0.76 & 0.70 & 2.64 & 3.13 & 2.08 & 2.59 & 0.76 & 2.64 & 1.98 & 1.95 \\
\hline Biochemistry \& Molecular Biology & 7.51 & 1.31 & 0.96 & 1.19 & 0.86 & 1.14 & 0.85 & 0.84 & 1.64 & 1.43 & 1.55 & 1.74 & 0.85 & 2.01 & 1.37 & 1.70 \\
\hline Developmental Biology & 7.00 & 1.30 & 0.91 & 1.29 & 0.66 & 1.09 & 0.85 & 0.61 & 3.40 & 3.55 & 2.82 & 3.65 & 0.52 & 3.52 & 2.71 & 1.90 \\
\hline Immunology & 6.83 & 1.30 & 0.93 & 1.10 & 0.90 & 0.97 & 0.85 & 0.96 & 2.01 & 1.85 & 1.78 & 1.94 & 0.85 & 2.16 & 1.68 & 1.98 \\
\hline Virology & 6.28 & 1.34 & 0.98 & 1.21 & 0.87 & 0.85 & 0.93 & 0.96 & 2.63 & 2.79 & 2.39 & 2.64 & 0.79 & 2.06 & 2.43 & 2.44 \\
\hline Hematology & 6.11 & 1.32 & 0.80 & 0.90 & 0.80 & 0.91 & 1.09 & 1.14 & 2.26 & 2.26 & 1.63 & 1.73 & 0.72 & 2.10 & 2.49 & 2.67 \\
\hline Infectious Diseases & 5.55 & 1.14 & 0.89 & 0.87 & 1.34 & 0.81 & 1.12 & 1.31 & 2.50 & 2.10 & 2.07 & 1.84 & 1.40 & 1.99 & 2.79 & 3.33 \\
\hline Genetics \& Heredity & 5.51 & 1.35 & 1.03 & 1.20 & 1.14 & 1.16 & 1.02 & 0.93 & 1.81 & 1.86 & 1.74 & 1.82 & 1.08 & 2.17 & 1.72 & 1.67 \\
\hline Biophysics & 5.42 & 1.10 & 1.05 & 1.27 & 0.91 & 1.14 & 1.02 & 1.04 & 1.72 & 1.46 & 1.71 & 2.01 & 0.82 & 1.94 & 1.74 & 2.00 \\
\hline Medicine Research \& Experimental & 5.22 & 1.66 & 0.90 & 1.15 & 0.96 & 1.03 & 1.22 & 1.02 & 1.81 & 2.30 & 1.63 & 2.03 & 0.99 & 2.15 & 2.36 & 2.03 \\
\hline Endocrinology \& Metabolism & 5.04 & 1.29 & 0.88 & 1.05 & 0.80 & 0.79 & 1.14 & 1.20 & 1.68 & 1.63 & 1.37 & 1.47 & 0.74 & 1.46 & 1.79 & 2.01 \\
\hline Physics, Atomic Molecular \& Chemical & 5.00 & 1.31 & 0.96 & 0.98 & 0.88 & 1.08 & 0.94 & 0.97 & 1.67 & 1.71 & 1.47 & 1.37 & 0.80 & 1.65 & 1.44 & 1.75 \\
\hline Neurosciences & 4.94 & 1.16 & 1.00 & 1.19 & 1.03 & 0.92 & 1.01 & 0.92 & 1.33 & 1.06 & 1.28 & 1.38 & 1.04 & 1.30 & 1.23 & 1.37 \\
\hline Physics, Nuclear & 4.78 & 1.45 & 1.12 & 1.15 & 1.31 & 1.08 & 0.91 & 0.75 & 1.90 & 2.36 & 1.94 & 2.10 & 1.14 & 1.69 & 1.77 & 1.56 \\
\hline Clinical Neurology & 4.77 & 1.24 & 0.82 & 0.94 & 0.68 & 1.02 & 1.00 & 1.07 & 2.00 & 1.87 & 1.52 & 1.62 & 0.62 & 2.19 & 1.75 & 2.13 \\
\hline Oncology & 4.71 & 1.21 & 1.00 & 1.24 & 1.04 & 0.92 & 1.17 & 1.12 & 1.44 & 1.24 & 1.38 & 1.60 & 1.03 & 1.41 & 1.74 & 1.86 \\
\hline Astronomy \& Astrophysics & 4.61 & 1.39 & 1.00 & 1.21 & 0.99 & 1.01 & 1.18 & 1.15 & 1.56 & 1.57 & 1.46 & 1.57 & 0.92 & 1.49 & 1.76 & 1.76 \\
\hline Physiology & 4.59 & 1.27 & 0.98 & 1.15 & 0.91 & 1.20 & 0.94 & 0.83 & 1.45 & 1.32 & 1.42 & 1.53 & 0.94 & 1.91 & 1.14 & 1.20 \\
\hline Physics & 4.55 & 1.85 & 1.17 & 1.20 & 1.44 & 1.37 & 1.00 & 0.74 & 1.23 & 1.88 & 1.40 & 1.43 & 1.36 & 1.67 & 1.30 & 1.14 \\
\hline Number of higest values (out of 152 fiel & & 70 & $\mathbf{0}$ & 26 & 12 & 10 & 15 & 24 & $\mathbf{n} / \mathbf{a}$ & 16 & 4 & 20 & 35 & 35 & 14 & 33 \\
\hline
\end{tabular}

Note: highest RCI and ARCI values in a field are bold Data Source: ISI data purchase 
Let us consider another example based on the second case, that is the case that examined the relationship between recognition and size across various national science. Table 7 gives the RCI, the rank by RCI and ARCI for six ISI Current Contents fields. The table is based on 1994-1998 citations and publications taken from ISI's NSIOD for the twenty largest publishing nations in each field. The RCI rank is determined only among the twenty countries.

In physics we see that Switzerland is ranked $1^{\text {st }}$ by both measures while the US drops from $2^{\text {nd }}$ to $8^{\text {th }}$. In biochemistry $\&$ biophysics the US drops from $1^{\text {st }}$ rank to $12^{\text {th }}$, the UK drops from $4^{\text {th }}$ to $10^{\text {th }}$ while Denmark and Belgium move from $9^{\text {th }}$ and $10^{\text {th }}$ to $3^{\text {rd }}$ and $4^{\text {th }}$, respectively. In fact, in every instance the US loses rank. In 5 of the 6 fields the UK's ARCI surpasses the US's ARCI compared to the picture portrayed using the RCI where the US dominated the UK in 5 of the 6 fields.

\section{Scale-independent indicators}

A power law relationship between a pair of variables is given by

$$
\mathrm{y}=\mathrm{kx}^{\mathrm{n}}
$$

where $\mathrm{n}$ is the slope of the $\log$-log regression line and $\mathrm{k}$ is a constant. Using this relationship we can calculate the expected $\mathrm{y}$-value, $\mathrm{y}_{\mathrm{e}}$, for a given $\mathrm{x}$ and $\mathrm{n}$. Now using the observed $\mathrm{y}-$ value, $\mathrm{y}_{\mathrm{o}}$, for the given $\mathrm{x}$ we can calculate a scale-independent indicator (SI) that is given by:

$$
\mathrm{SI}(\mathrm{x}, \mathrm{n})=\mathrm{y}_{\mathrm{o}} / \mathrm{y}_{\mathrm{e}}
$$

Let us explore how the UK science system looks within the context of other OECD sciences system and compared to how it is portrayed using various SI indicators compared to traditional indicators such as RCI.

Table 8 examines the relationship between size \& HERD ${ }^{23}$, recognition \& HERD, size \& researchers ${ }^{24}$, and recognition $\&$ size. It is important to note that in this instance size (i.e. number of papers) includes counts of research papers in the sciences, the social sciences and arts \& humanities ${ }^{25}$. The focus of this paper is not an evaluation of science systems but rather an illustration of how to develop scale-independent indicators. The OECD countries in Table 8 are listed in decreasing order of SI value. The traditional indicator is determined by simply taking the ratio between the two variables. For example the first set of columns explore the relationship between size and HERD. The first column within the set gives the OECD country name, the second column the SI and the third column the ratio of size and HERD (i.e. papers/\$HERD). The value in the brackets in the third column is the rank determined using the ratio. The exponent for the power law relationship and the coefficient of determination $\left(\mathrm{R}^{2}\right)$ for the pair of variables is given at the top of the list. For example, the exponent for the power law relationship between 1994-98 papers and $1995 \mathrm{HERD}^{26}$ is $0.83 \pm 0.06^{27}$ and it has $\mathrm{a} \mathrm{R}^{2}=0.88$.

\footnotetext{
${ }^{23}$ HERD is the Higher Education Expenditure on R\&D in million current PPP dollars.

24 The OECD defines a researcher as a university graduate. This is not a particularly satisfactory definition of a 'researcher' but it probably as good a measure as using population values like May (1997) used.

25 Unfortunately ISI's NSIOD does not provide aggregate individual counts for the sciences, social sciences and arts \& humanities that are readily accessible from the data set. Total counts for all three areas combined and for individual CC fields are more accessible.

${ }^{26}$ For a few countries the 1995 values were not available so the most recent values were used.

27 A similar analysis was done using 1995 scientific and technical paper and 1995 GDP (\$US) for 48 countries. The exponent was found to be $0.85 \pm 0.08$ and $R^{2}=0.72$. Data source: National Science Board's Science \& Engineering Indicators, 1998, Table 5-50.
} 


\begin{tabular}{|c|c|c|c|c|c|c|c|c|c|c|c|}
\hline \multicolumn{4}{|c|}{ Physics } & \multicolumn{4}{|c|}{ Biochemistry \& Biophysics } & \multicolumn{4}{|c|}{ Molecular Biology \& Genetics } \\
\hline Country & RCI & Rank & ARCI & Country & RCI & Rank & ARCI & Country & RCI & Rank & ARCI \\
\hline 1 Switzerland & 1.89 & 1 & 1.96 & Switzerland & 1.32 & 2 & 1.89 & Finland & 1.27 & 3 & 1.72 \\
\hline 2 Israel & 1.43 & 3 & 1.63 & Israel & 1.05 & 5 & 1.69 & Switzerland & 1.41 & 1 & 1.65 \\
\hline 3 Netherlands & 1.37 & 4 & 1.57 & Denmark & 0.99 & 9 & 1.65 & Austria & 1.10 & 9 & 1.61 \\
\hline 4 Canada & 1.30 & 7 & 1.35 & Belgium & 0.95 & 10 & 1.52 & Israel & 1.08 & 10 & 1.41 \\
\hline 5 Sweden & 1.14 & 9 & 1.34 & Austria & 0.83 & 13 & 1.47 & Netherlands & 1.20 & 6 & 1.32 \\
\hline 6 UK & 1.33 & 5 & 1.21 & Netherlands & 1.00 & 8 & 1.42 & Belgium & 0.98 & 11 & 1.23 \\
\hline 7 Spain & 1.09 & 11 & 1.17 & Sweden & 1.00 & 7 & 1.40 & Canada & 1.17 & 7 & 1.17 \\
\hline 8 US & 1.64 & 2 & 1.17 & Canada & 1.12 & 3 & 1.39 & Sweden & 0.96 & 12 & 1.15 \\
\hline 9 Australia & 1.00 & 12 & 1.16 & Australia & 0.86 & 12 & 1.28 & France & 1.21 & 5 & 1.13 \\
\hline 10 Germany & 1.31 & 6 & 1.07 & UK & 1.07 & 4 & 1.15 & Denmark & 0.86 & 15 & 1.13 \\
\hline 11 France & 1.18 & 8 & 1.03 & Germany & 1.02 & 6 & 1.11 & UK & 1.27 & 4 & 1.10 \\
\hline 12 Italy & 1.13 & 10 & 1.03 & US & 1.36 & 1 & 1.05 & Germany & 1.13 & 8 & 1.04 \\
\hline 13 Poland & 0.87 & 14 & 0.92 & France & 0.90 & 11 & 1.03 & Australia & 0.87 & 14 & 0.99 \\
\hline 14 Japan & 0.97 & 13 & 0.85 & Spain & 0.61 & 16 & 0.87 & Italy & 0.89 & 13 & 0.95 \\
\hline 15: Brazil & 0.66 & 16 & 0.75 & Italy & 0.64 & 15 & 0.82 & US & 1.31 & 2 & 0.89 \\
\hline 16 India & 0.59 & 17 & 0.61 & Japan & 0.77 & 14 & 0.76 & Japan & 0.81 & 16 & 0.76 \\
\hline 17 South Korea & 0.48 & 18 & 0.56 & Poland & 0.41 & 17 & 0.68 & Spain & 0.64 & 17 & 0.75 \\
\hline 18 Russia & 0.66 & 15 & 0.56 & Russia & 0.36 & 18 & 0.49 & India & 0.27 & 19 & 0.38 \\
\hline 19 China/Hong Kong & 0.39 & 19 & 0.38 & South Korea & 0.29 & 19 & 0.49 & Brazil & 0.29 & 18 & 0.38 \\
\hline 20 Peoples R China & 0.38 & 20 & 0.37 & India & 0.25 & 20 & 0.39 & Russia & 0.21 & 20 & 0.22 \\
\hline
\end{tabular}

\begin{tabular}{|c|c|c|c|c|c|c|c|c|c|c|c|}
\hline \multicolumn{4}{|c|}{ Electrical \& Electronic Engineering } & \multicolumn{4}{|c|}{ Biotechnol \& Applied Microbiology } & \multicolumn{4}{|c|}{ Pharmacology \& Toxicology } \\
\hline Country & RCI & Rank & ARCI & Country & RCI & Rank & ARCI & Country & RCI & Rank & $\overline{\text { ARCI }}$ \\
\hline 1 Sweden & 1.39 & 1 & 1.86 & Switzerland & 1.91 & 1 & 2.40 & Switzerland & 1.76 & 1 & 2.38 \\
\hline 2 Netherlands & 1.31 & 4 & 1.71 & Belgium & 1.63 & 4 & 2.16 & Sweden & 1.37 & 3 & 1.76 \\
\hline 3 Belgium & 1.12 & 6 & 1.51 & Sweden & 1.65 & 2 & 2.08 & UK & 1.52 & 2 & 1.61 \\
\hline Australia & 1.20 & 5 & 1.50 & Netherlands & 1.64 & 3 & 1.88 & Finland & 1.08 & 7 & 1.55 \\
\hline 5 Germany & 1.32 & 3 & 1.43 & Australia & 1.06 & 10 & 1.33 & Netherlands & 1.13 & 6 & 1.44 \\
\hline 6 France & 1.11 & 7 & 1.24 & Canada & 1.24 & 8 & 1.29 & Belgium & 1.04 & 8 & 1.43 \\
\hline 7 Russia & 0.92 & 10 & 1.18 & Italy & 1.09 & 9 & 1.28 & Canada & 1.19 & 5 & 1.41 \\
\hline 8 Spain & 0.92 & 11 & 1.17 & Germany & 1.29 & 7 & 1.28 & Australia & 1.01 & 10 & 1.30 \\
\hline 9 US & 1.32 & 2 & 1.11 & UK & 1.30 & 6 & 1.21 & US & 1.35 & 4 & 1.20 \\
\hline 10 UK & 1.04 & 8 & 1.05 & US & 1.38 & 5 & 1.12 & France & 1.01 & 9 & 1.14 \\
\hline 11 Canada & 0.93 & 9 & 1.02 & Spain & 0.77 & 11 & 0.83 & Germany & 0.92 & 11 & 1.01 \\
\hline 12 Hong Kong & 0.72 & 15 & 0.95 & South Korea & 0.70 & 14 & 0.80 & Italy & 0.83 & 12 & 0.95 \\
\hline 13 Italy & 0.84 & 13 & 0.95 & Taiwan & 0.61 & 15 & 0.78 & Taiwan & 0.63 & 15 & 0.89 \\
\hline 14Taiwan & 0.82 & 14 & 0.92 & France & 0.72 & 13 & 0.71 & Spain & 0.65 & 14 & 0.82 \\
\hline 15 Japan & 0.89 & 12 & 0.84 & Japan & 0.76 & 12 & 0.68 & Brazil & 0.55 & 16 & 0.79 \\
\hline 16! Singapore & 0.55 & 17 & 0.72 & Brazil & 0.48 & 16 & 0.61 & Japan & 0.74 & 13 & 0.75 \\
\hline 17 South Korea & 0.59 & 16 & 0.70 & Czech Republic & 0.47 & 17 & 0.61 & South Korea & 0.37 & 17 & 0.52 \\
\hline 18 China/Hong Kong & 0.54 & 18 & 0.62 & China/Hong Kong & 0.42 & 18 & 0.52 & China/Hong Kong & 0.36 & 18 & 0.48 \\
\hline 19Peoples R China & 0.44 & 19 & 0.54 & Peoples R China & 0.36 & 20 & 0.47 & Peoples R China & 0.31 & 19 & 0.43 \\
\hline 20 India & 0.31 & 20 & 0.38 & India & 0.42 & 19 & 0.43 & India & 0.30 & 20 & 0.41 \\
\hline
\end{tabular}

Rank - rank by RCI; data source: ISI National Science Indicators on Diskette 1981-1998 
Table 8 - Various scale-independent ( SD) and ratio indicators for OECD countries

\begin{tabular}{|c|c|c|c|c|c|c|c|c|c|c|c|}
\hline \multicolumn{3}{|c|}{$\operatorname{Size}^{1}(P) \&$ HERD $^{2}($ PPP \$M) } & \multicolumn{3}{|c|}{ Recognition $^{1}$ (C) \& HERD ${ }^{2}$ (PPP \$M } & \multicolumn{3}{|c|}{$\operatorname{Size}^{1}(\mathbf{P}) \&$ Researchers $^{3}(\mathbf{R})(1995)$} & \multicolumn{3}{|c|}{$\operatorname{Recognition}^{1}(\mathrm{C}) \& \operatorname{Size}^{1}(\mathrm{P})$} \\
\hline \multicolumn{2}{|l|}{$\mathrm{n}=0.83 \pm 0.06$} & \multirow{2}{*}{$\begin{array}{c}R^{2}=0.88 \\
P / \$ M\end{array}$} & \multicolumn{2}{|c|}{$\mathrm{n}=0.90 \pm 0.09$} & \multirow{2}{*}{$\begin{array}{c}\mathbf{R}^{2}=0.81 \\
C / \$ M\end{array}$} & \multicolumn{2}{|c|}{$\mathrm{n}=0.79 \pm 0.12$} & \multirow{2}{*}{$\begin{array}{c}\mathbf{R}^{2}=0.63 \\
\mathbf{P} / \mathbf{R}\end{array}$} & \multicolumn{2}{|c|}{$\mathrm{n}=1.11 \pm 0.05$} & \multirow{2}{*}{$\begin{array}{c}\mathrm{R}^{2}=0.96 \\
\mathrm{RCI}\end{array}$} \\
\hline Country & SI & & Country & SI & & Country & SI & & Country & SI & \\
\hline 1 Czech Republic & 2.26 & $36.7(1)$ & United Kingdom & 2.19 & $70.5(4)$ & United States & 2.90 & $9.2(12)$ & Iceland & 1.96 & $\overline{1.16(8)}$ \\
\hline 2 United Kingdom & 1.88 & $16.0(7)$ & Denmark & 1.90 & $76.7(1)$ & United Kingdon & 2.55 & $11.2(6)$ & Switzerland & 1.77 & $1.61(1)$ \\
\hline 3 New Zealand & 1.67 & $24.9(3)$ & United States & 1.84 & $49.0(11)$ & Sweden & 2.40 & $15.7(1)$ & Denmark & 1.46 & $1.25(4)$ \\
\hline 4 Hungary & 1.64 & $25.4(2)$ & Switzerland & 1.80 & $65.7(7)$ & Switzerland & 2.30 & $15.3(2)$ & Finland & 1.37 & $1.15(9)$ \\
\hline 5 Canada & 1.57 & $14.7(9)$ & Canada & 1.80 & $61.0(8)$ & Canada & 2.19 & $10.9(7)$ & Sweden & 1.34 & $1.24(5)$ \\
\hline 6 United States & 1.51 & $9.2(20)$ & New Zealand & 1.71 & 76.0 (2) & Netherlands & 1.96 & $11.3(5)$ & Netherlands & 1.34 & 1.28 \\
\hline 7 Poland & 1.41 & $17.9(4)$ & Finland & 1.66 & $67.2(5)$ & France & 1.87 & $8.4(14)$ & Belgium & 1.33 & $1.16(7)$ \\
\hline 8 Australia & 1.36 & $13.9(10)$ & Czech Republic & 1.61 & $75.2(3)$ & Australia & 1.84 & $10.2(9)$ & Austria & 1.17 & $0.98(14)$ \\
\hline 9 Denmark & 1.31 & $16.5(5)$ & Hungary & 1.46 & $66.3(6)$ & Germany & 1.76 & $7.2(16)$ & Norway & 1.17 & $0.95(15)$ \\
\hline 10 Finland & 1.23 & $15.6(8)$ & Sweden & 1.44 & $52.3(10)$ & Denmark & 1.66 & $11.8(4)$ & United States & 1.11 & $1.42(2)$ \\
\hline 11 Spain & 1.19 & $12.2(13)$ & Netherlands & 1.39 & $48.7(12)$ & Finland & 1.63 & $11.9(3)$ & Canada & 1.09 & $1.11(10)$ \\
\hline 12 France & 1.18 & $9.8(18)$ & Australia & 1.35 & $48.3(13)$ & Belgium & 1.52 & $9.9(10)$ & United Kingdom & 1.07 & $1.18(6)$ \\
\hline 13 Greece & 1.17 & $16.4(6)$ & France & 1.20 & $38.3(16)$ & New Zealand & 1.35 & $10.6(8)$ & Ireland & 1.06 & $0.79(20)$ \\
\hline 14 Sweden & 1.08 & $11.3(14)$ & Belgium & 1.11 & $41.3(14)$ & Norway & 1.28 & $9.5(11)$ & New Zealand & 1.02 & $0.82(18)$ \\
\hline 15 Netherlands & 1.04 & $10.3(16)$ & Germany & 1.05 & $31.9(22)$ & Japan & 1.23 & $4.5(20)$ & Italy & 1.01 & 1.01 (13) \\
\hline 16 Switzerland & 1.03 & $10.9(15)$ & Italy & 1.02 & $34.0(21)$ & Italy & 1.22 & $5.6(18)$ & Germany & 1.00 & 1.09 (11) \\
\hline 17 Germany & 1.03 & $7.9(22)$ & Spain & 1.02 & 36.2 (19) & Austria & 1.09 & $7.3(15)$ & France & 0.99 & $1.05(12)$ \\
\hline 18 Italy & 1.01 & $9.0(21)$ & Iceland & 1.00 & 54.6 (9) & Spain & 1.06 & $5.2(19)$ & Australia & 0.98 & $0.94(16)$ \\
\hline 19 Ireland & 0.88 & $12.9(11)$ & Poland & 0.97 & $39.4(15)$ & Ireland & 0.86 & $7.0(17)$ & Portugal & 0.91 & $0.67(22)$ \\
\hline 20 Belgium & 0.87 & 9.5 (19) & Norway & 0.88 & $35.1(20)$ & Iceland & 0.67 & $8.8(13)$ & Hungary & 0.90 & $0.70(21)$ \\
\hline 21 Norway & 0.80 & $10.0(17)$ & Ireland & 0.86 & $38.2(17)$ & Greece & 0.64 & $4.2(21)$ & Spain & 0.84 & 0.80 (19) \\
\hline 22 Austria & 0.63 & $7.1(24)$ & Greece & 0.85 & 36.5 (18) & Hungary & 0.60 & $4.0(22)$ & Japan & 0.80 & $0.87(17)$ \\
\hline 23 Iceland & 0.60 & $12.7(12)$ & Austria & 0.69 & $25.9(23)$ & Czech Republic & 0.54 & $3.4(23)$ & Greece & 0.75 & $0.60(23)$ \\
\hline 24 Japan & 0.54 & $3.6(25)$ & Portugal & 0.42 & $17.9(24)$ & Poland & 0.43 & $2.1(24)$ & Czech Republic & 0.70 & $0.55(25)$ \\
\hline 25 Portugal & 0.53 & $7.2(23)$ & Japan & 0.42 & $11.7(25)$ & Korea & 0.33 & $1.5(26)$ & Poland & 0.69 & $0.59(24)$ \\
\hline 26 Turkey & 0.30 & $3.3(26)$ & Mexico & 0.18 & $6.5(26)$ & Portugal & 0.29 & $1.8(25)$ & Mexico & 0.69 & $0.54(26)$ \\
\hline 27 Mexico & 0.30 & $3.2(27)$ & Turkey & 0.11 & $4.2(27)$ & Turkey & 0.15 & $0.7(27)$ & Korea & 0.54 & $0.46(27)$ \\
\hline 28 Korea & na & na & Korea & na & na & Mexico & 0.11 & $0.4(28)$ & Turkey & 0.44 & $0.34(28)$ \\
\hline
\end{tabular}

Key: P - refereed papers (article, note, review \& conference proceedings); C - citations counted with a variable window; $\mathrm{n}$ - power law exponent

1. Source: ISI 1981-1998 NSIOD - 1994-98 Science, Social Science and Arts \& Humanities papers

2. Source OECD, EAS (MSTI database), April 1997 Table 451995 or closest available year

3. Source: OECD, MSTI database, April 1999. Researcher or university graduate 
On average, it appears that when the expenditure on higher education is doubled the number of research papers produced only increases by 1.78 times. The fact that the measure of size includes papers in the social sciences and the arts \& humanities may contribute to the exponent being less than 1.0. Also, factors such as increasing overhead and infrastructure costs or even the idea that larger countries tend to contribute more to 'big' science projects than smaller countries could contribute to publishing size not increasing as rapidly as HERD. However, given the evidence that the exponent is less than 1.0 we can see that the Czech Republic has the highest rank determined by both the SI and ratio indicators. On the other hand the UK is ranked $2^{\text {nd }}$ by SI while it only ranked $7^{\text {th }}$ using the ratio indicator. In other words according to the SI the UK is doing quite well when its publishing size is adjusted in light of the evidence that the amount of publishing seems to decrease with HERD across OECD public research systems.

Examining the other indicators we see that the UK has a higher rank according to the SI for recognition \& HERD and size \& researchers than it has using the ratio indicator. In the case of recognition \& size while the SI indicator ranks the UK lower than the ratio indicator (i.e. in this instance the RCI) the gap between the US and the UK is significantly lower.

A glimpse at the lower ranked countries in Table 8 indicates that the SI indicator does not automatically promote small countries to higher ranks. In fact May (1997, p. 794) made the observation about his data ".. that there is no high relative performance by a very small country. This is understandable; science has a threshold of critical size and investment."

Finally, these findings suggest that bibliometric techniques that use statistical methods to assign papers and citations derived in one classification scheme to another classification scheme may be acutely misleading. For example the Adams (1998) study assigned statistically weighted proportions of ISI's Current Contents (CC) journal papers and citations to each research assessment exercise (RAE) unit of assessment (UOA). The weighting was determined by statistically examining the mapping between journals named in the list of research outputs submitted to the 1996 RAE and the ISI's Current Contents and did not take into account the power law relationship between papers and citations. This probably produced erroneous indicators and resulted in misleading conclusions. Only through meticulous attention to detail can we build robust indicators that will properly reflect the accuracy of the information embedded in data sets like ISI's citation indices.

Some investigators may wish to attribute the non-linear relationship between recognition and size to the process of self-recognition. In other words, individuals citing their own work, colleagues citing colleagues or countryman citing countryman preferentially over those from other nations. Even if this is the case it does not negate the fact that traditional impact or RCI measures are inaccurate. Citing previous work, be it one's own work or the work of others, is intrinsic to the activity of knowledge production. In fact disseminating ideas via an 'invisible college' [Price et al., 1966] and other social networks is an effect way to transfer ideas. It is an admired and valued activity. Perhaps we could try to adjust for excessive self-citations, if we could define 'excessive'. But can we justify adjusting citation counts to account for geographical and social proximity [Katz and Martin, 1997]? In fact, it is quite likely that these adjustments would not remove the power law relationship between recognition and size. It certainly would not affect the power law relationship between size and expenditure or between size and numbers of researchers. Perhaps we should ask ourselves if bibliometric indicators are fundamentally flawed or are they merely a reflection of the universal nature of scale-independent characteristics that may be inherent to many human activities?

\section{Conclusions}

The existence of power law distributions in some human activity has been known for nearly a century. In fact, the Pareto (1897) income distribution is one of the earliest social power laws to have been found. Scale-independent relationships have also been noted in how we use 
words (Zipf, 1949) and the productivity of researchers (Lotka, 1926). In fact, over the past 20 years a countless number of power laws have been observed in human processes ranging from the distribution of company sizes and the fluctuations in stock prices (Mandelbrot 1997) to the flow of messages on the internet (Crovella et al, 1997) and movement of traffic on our highways (Buchanan, 1997).

In research evaluation activities that go back past the landmark work of de Solla Price (1963) it has been noted that 'skewed' or power law distributions characterise many of the activities in the scientific community. However, little effort has been made to account for the non-linear impact that can arise from these distributions when comparing the research performance of groups, institutions and nations of differing size.

This paper has demonstrated that power law relationships can be found between recognition, impact and collaboration and publishing size. It has also shown that there are power law relationships between publishing size and R\&D expenditure or number of researchers. The exponent of the power law is sometimes greater than 1.0 indicating the existence of a "Matthew effect". Other times it is less than 1.0 indicating an "inverse Matthew effect".

Once a power law has between observed between two variables the exponent can be used to calculate a scale-independent indicator based on the ratio of the observed value of the dependent variable to the expected average value of the dependent variable for a given value of the independent variable. The use of scale-independent indicators in the evaluation of research performance might significantly change our understanding of the landscape of the relationship among participants in the research system. It may also help us account for the intricate dynamics of the national and international innovation systems.

\section{What next?}

Before suggesting a research agenda to explore the potential for further research in scaleindependent indicators here is an important warning. The scale-independent indicators that have been prepared in different sections of this paper are not comparable. For example sometimes citations were counted using a fixed citation window and other times they were counted using a variable citation window. In some instances papers were classified using ISI's overlapping subject categories or ISI's Current Contents journal. And finally sometimes papers were counted over a fourteen-year time interval while at other times they were counted using a five-year time interval.

Perhaps the first item on the research agenda should be to do a more rigorous comparison of the relationship between recognition and impact and size (a) across scientific fields within national science systems and (b) across national science systems within scientific fields using the traditional RCI indicator and the scale-independent ARCI indicator. Refereed papers should be classified into scientific fields using one scheme. Perhaps ISI's overlapping subject categories scheme might be the best because it has some interesting advantages that will not be discussed here. Citations should be counted using a 3, 4 and 5 year fixed citation window to reduce the affect of the varying rate at which citations accumulate using the variable citation window. This will allow us to investigate how the scale-independent nature of recognition varies with the width of the citation window. Also, counting papers and citations using a fixed window on an annual basis will facilitate time studies.

The second study on the research agenda might be to repeat the first study but restricting the analysis to internationally co-authored papers. This could facilitate a better understanding of the scale-independent relationship between the amount of international collaboration, and probably the impact of collaborative research, and the size of a science system.

Finally the scale-independent relationships recognition, impact, collaboration and size with other demographic and economic variables should be investigated. 
Perhaps by building robust scale-independent indicators we might be able to gain a deeper understanding of the role that chaos theory and complexity play in the self-organising structures that seem to emerge from dynamic human activity. It might even help us to understand how to formulate scale-independent policies that might facilitate a reduction in some of the inequities that are found in society and yet nurture the evolution of other valuable scale-independent social processes. However, before we can do that we may need to construct many more robust social and economic data sets like ISI's citation indices to help us encapsulate the signature of dynamic human processes.

\section{Acknowledgments:}

I would like to thank my father, Leon Katz, for the many years he has spent with me exploring wonders of nonlinear systems and the importance of power laws. Special appreciation is given to David Hamilton from DERA who reproduced many of my calculations and pointed out some interesting problems. As always Diana Hicks made insightful suggestions that contributed to improving the tone of the paper. Linda Butler confirmed that the SI indicators for Australia seem realistic. Comments by Keith Pavitt and Francis Narin on an earlier version were encouraging.

\section{References}

1. Adams J, Bailey T, Jackson L, Scott P, Pendlebury D and Small H (1998). Benchmarking of the international standing of research in England, Report of a consultancy study on bibliometric analysis prepared for the Higher Education Funding Council of England, Center for Policy Studies, University of Leeds and the Institute for Scientific Information. (available at http://www.leeds.ac.uk/benchmark/)

2. Barenblatt G.L. (1997) Scaling, Self-similarity, and Intermediate Assumptions (Cambridge Univ. Press.)

3. Buchanan M (1997), One law to rule them all, New Scientist, November 8, 1997 (available at http://www.newscientist.com/ns/971108/features.html)

4. Crovella ME and Bestavros A (1997). "Self-Similarity in World Wide Web Traffic: Evidence and Possible Causes," in IEEE/ACM Transactions on Networking, 5(6):835846, December 1997

5. May RM (1997). The scientific wealth of nations, Science, 275, 793-796 (available at http://sowi.iwp.uni-linz.ac.at/dialog/97swn/)

6. Katz JS (1999a). The self-similar science system, Research Policy, 28, 501-517 (available at http://www.sussex.ac.uk/spru/jskatz)

7. Katz JS (1999b). Bibliometric indicators and the social sciences, prepared for the ESRC, (available at http://www.sussex.ac.uk/spru/jskatz)

8. Katz JS and Katz L (1999). Power laws and athletic performance, J. Sport Sciences, 17, pp. 467-476 (available at http://www.sussex.ac.uk/spru/jskatz).

9. Katz JS and Hicks D (1997a), The changing shape of British Industrial research, STEEP Special Report No. 6, SPRU, University of Sussex, Brighton, BN1 9RF, UK.

10. Katz JS and Hicks DM (1997b). Bibliometric Indicators for National Systems of Innovation prepared for IDEA project funded by TSER program of the EC, ESRC STEEP Centre, Science Policy Research Unit, University of Sussex, Brighton, BN1 9RF, UK (available at http://www.sussex.ac.uk/spru/jskatz)

11. Katz JS and Martin BR (1997). What is Research Collaboration, Research Policy, 26, pp 1-18 (available at http://www.sussex.ac.uk/spru/jskatz)

12. Katz JS and Hicks DM (1995). The Classification of Interdisciplinary Journals: A New Approach, Proceedings of the Fifth Biennial Conference of the International Society for Scientometrics and Infometrics, Rosary College, ILL, Medford NJ: Learned Information Inc., pp. 245-255.

13. Lotka AJ (1926). The frequency distribution of scientific productivity, J. Wash Acad. Sci.16, 317-323 
14. Merton RK (1968). The Matthew Effect in Science, Science, 159, 3810, pp 56-63 (available at http://www.garfield.library.upenn.edu/merton/list.html)

15. Mandelbrot BB (1997), Fractals and Scaling in Finance : Discontinuity, Concentration, Risk (Springer-Verlag, Nov 1997)

16. Pareto V (1897). Cours d' économie politique 2, 299-345.

17. De Solla Price DJ (1963). Little Science, Big Science, New York: Columbia Press.

18. Price DJ de Solla Price and D deB. Beaver (1966). Collaboration in an Invisible College, American Psychologist 21, pp. 1011-18

19. Zipf GK (1949). Human Behavior and the Principle of Least Effort, New York: EdisonWesley, 24-25 